\title{
Line Transversals to Translates of a Convex Body
}

\author{
J. Jerónimo-Castro • E. Roldán-Pensado
}

Received: 30 December 2009 / Revised: 5 June 2010 / Accepted: 25 August 2010 /

Published online: 17 September 2010

(C) Springer Science+Business Media, LLC 2010

\begin{abstract}
Let $K$ be a convex body in the plane. Define $\lambda(K, t)$ as the smallest number satisfying the following: if $\mathcal{F}$ is any family of translates of $K$ such that every $t$ members of $\mathcal{F}$ have a common transversal, then all the members of $\lambda(K, t) \mathcal{F}$ have a common transversal. We give bounds for $\lambda(K, 3)$ and $\lambda(K, 4)$ for a general convex figure $K$. In particular, we obtain that $\lambda(K, 3) \leq 1.79$ when $K$ is the Euclidean disc.
\end{abstract}

Keywords Line transversal . Translates - Convex body

\section{Introduction}

Let $\mathcal{F}$ be a family of compact convex bodies in the plane. We say that $\mathcal{F}$ has the property $T$ if there exists a line that intersects every member of $\mathcal{F}$. Also, if $k$ is a given positive integer we say that $\mathcal{F}$ has the property $T(k)$ if any subfamily of $\mathcal{F}$ with $k$ members has the property $T$. Let $K$ be a convex body in the plane, $\mathcal{F}=\left\{x_{1}+K\right.$, $\left.\ldots, x_{n}+K\right\}$ be a finite family of translates of $K$, and $\lambda>0$, then the family $\lambda \mathcal{F}$ is defined as $\left\{x_{1}+\lambda K, \ldots, x_{n}+\lambda K\right\}$.

Given a centrally symmetric convex body $K$ in the plane, we denote by $\mathrm{d}_{K}$ the Minkowski distance (or $K$-distance), that is, the distance function associated with the body $K$ in the usual manner: given a point $x \in \mathbb{R}^{2}$ we define the $K$-norm

$$
\|\cdot\|_{K}: \mathbb{R}^{2} \rightarrow \mathbb{R}
$$

Research supported by the Alfréd Rényi Mathematics Institute.

J. Jerónimo-Castro (凶)

Facultad de Ingenieria, Universidad de Queretaro, Av. del Gran Cue 18 int. 21-B Col. El Pueblito

Corregidora, Queretaro, Mexico, CP 62400

e-mail: jeronimo@ cimat.mx

E. Roldán-Pensado

Hule 9 Ejido de Acapantzingo Cuernavaca, Morelos, Mexico, CP 62440

e-mail: edgardo@matcuer.unam.mx 
Fig. 1 Geometric meaning of the $K$-norm

Fig. $2 K$-width of $X$ in direction $u$
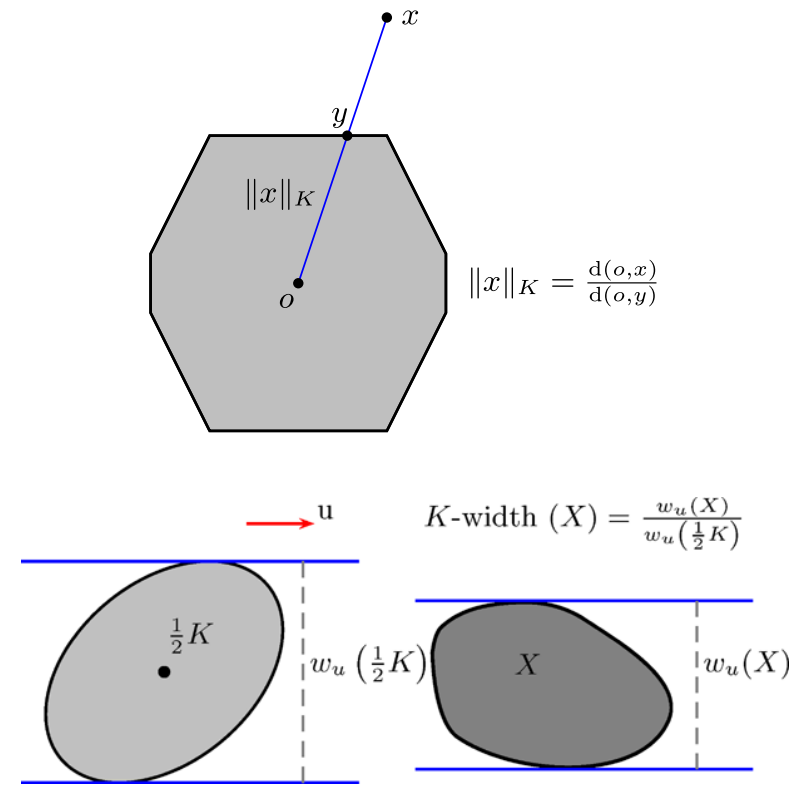

as $\|x\|_{K}=\inf \left\{\lambda \in \mathbb{R}^{+}: x \in \lambda K\right\}$. Furthermore, this norm defines a metric as follows: $\forall x, y \in \mathbb{R}^{2}$ we define the $K$-distance between points $x$ and $y$ as $\mathrm{d}_{K}(x, y)=\|x-y\|_{K}$.

Intuitively (see Fig. 1), the geometric meaning of the $K$-norm of a given point $x \in \mathbb{R}^{2}$ is the following: let $y$ be the point where the ray $\overrightarrow{o x}$ intersects the boundary of $K$, then

$$
\|x\|_{K}=\frac{\mathrm{d}(o, x)}{\mathrm{d}(o, y)},
$$

where $\mathrm{d}(z, w)$ stands for the Euclidean distance between the points $z$ and $w$. Also, given a set $X$ in the plane we define the $K$-width of $X$ in direction $u$ as $\frac{w_{u}(X)}{w_{u}\left(\frac{1}{2} K\right)}$, where $w_{u}(Y)$ denotes the Euclidean width of a set $Y$ in direction $u$ (see Fig. 2). Furthermore, the $K$-width of $X$ is defined as

$$
\inf \left\{\frac{w_{u}(X)}{w_{u}\left(\frac{1}{2} K\right)}: u \in \mathbb{S}^{1}\right\} .
$$

In 1964 B. Grünbaum [4] raised the following question:

Let $K$ be a convex body in the plane. What is the smallest positive number $\lambda=$ $\lambda(K, s)$ such that if $\mathcal{F}$ is any finite family of translates of $K$ with the property $T(s)$, then the family $\lambda \mathcal{F}$ has the property $T$ ?

It seems that the problem is not easier if we consider only families of pairwise disjoint translates, so it is well justified to define the number $\lambda_{\text {disj }}(K, s)$ in an analogous way for disjoint families of translates of a convex body $K$.

Some results on Grünbaum's problem are listed below. The first two of them are due to J. Eckhoff [2]: 
Theorem $\mathbf{E 1}$ Let $K$ be a convex body in the plane, then

$$
\lambda(K, 3) \leq 2 .
$$

Theorem E2 Let $Q$ be the unit square, then

$$
\lambda(Q, 3)=2 .
$$

In what follows we will denote the unit disc by $B$. For the case of the disc, A. Heppes [5] proved the following:

Theorem H1 $\lambda_{\operatorname{disj}}(B, 3)<1.65$.

And the first named author [8] of this work proved:

Theorem J $\lambda(B, 4)=\frac{1+\sqrt{5}}{2}$.

There also are some asymptotic results, for instance [6]:

Theorem H2 For every real number $w \in(0,1]$ there is a number $N(w)$ such that for every $T(3)$-family, $\mathcal{F}$, of at least $N(w)$ pairwise disjoint translates of a convex figure $K$ we have that $(1+w / 2) \mathcal{F}$ has the property $T$.

In this paper we give bounds of $\lambda(K, 3)$ and $\lambda(K, 4)$ for arbitrary convex bodies. We also give a proof of the following fact observed by J. Eckhoff [2]: $\lambda(K, 3)=2$ if and only if $K$ is a parallelogram. As far as we know, a proof of this result has never been published.

\section{New Results}

We begin with some interesting and useful comments. By an useful reduction noticed by H. Tverberg [11] we know that $K$ and $\frac{1}{2}(K-K)$ share the same properties with respect to line transversals. So, in what follows we consider only translates of a centrally symmetric convex body $K$. Let us assume that $K$ is centered at the origin $O$. It is not difficult to see that if three translates $K+a, K+b, K+c$ have a common line transversal $\ell$ intersecting them in that order, then the line $\ell^{\prime}$ parallel to $[b, c]$ and equidistant from the points $a, b, c$, also intersects the translates in the same order. To see this proceed as follows: start to shrink (continuously and uniformly) the translates until they have no longer a line transversal intersecting them in that order. Consider the last moment they still having a transversal in the given order, then the transversal is tangent to each one of the shrunk translates $\alpha K+a, \alpha K+b, \alpha K+c$.

The Banach-Mazur distance between two centrally symmetric convex bodies $K_{1}$ and $K_{2}$ centered at the origin is the smallest positive number $\delta=\delta\left(K_{1}, K_{2}\right)$ such that there exists a linear transformation $\varphi$ that satisfies $K_{1} \subset \varphi\left(K_{2}\right) \subset \delta K_{1}$. The application $\delta\left(K_{1}, K_{2}\right)$ is a multiplicative metric called the Banach-Mazur metric. 
Fig. 3 Line transversal to three translates

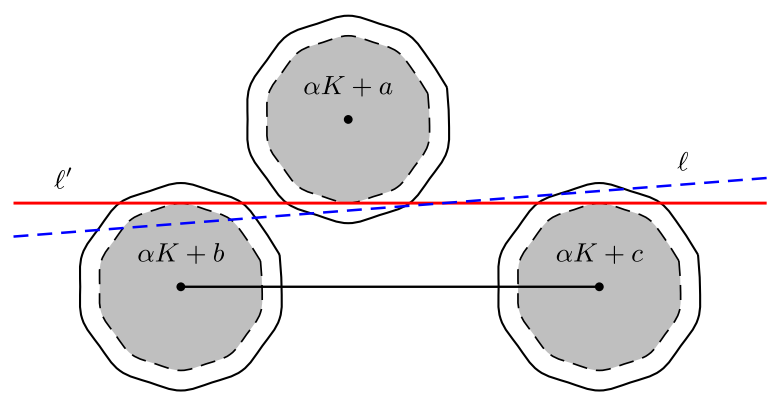

Now consider the equivalence relationship: $K_{1} \sim K_{2}$ if there exists an affine transformation $\varphi$ such that $\varphi\left(K_{1}\right)=K_{2}$. The quotient space of the set of centrally symmetric convex bodies, with this equivalence relationship, is called the Banach-Mazur compactum. This name is justified by the fact that, together with $\delta$, this space is a compact metric set. It is well known (see [1]) that the Banach-Mazur compactum is homeomorphic to the Alexandroff compactification of a Hilbert manifold.

It is not difficult to see that for a fixed $r$, the application $[K] \mapsto \lambda(K, r)$, where $[K]$ is the class of sets affinely equivalent to $K$, is well defined and continuous on the Banach-Mazur compactum. We have, in fact, a little more than continuity.

Lemma 1 If $K_{1}$ and $K_{2}$ are centrally symmetric convex bodies, then

$$
\lambda\left(K_{1}, r\right) \leq \lambda\left(K_{2}, r\right) \delta\left(K_{1}, K_{2}\right) .
$$

For one of the main results, we need some additional definitions. Let $X$ be a set of points in the plane, we say that $X$ has the property $T_{K}$ if the $K$-width of $X$ is at most 1 . If $r$ is a positive integer, we say that $X$ has the property $T_{K}(r)$ if any subset of $X$ with $r$ points has the property $T_{K}$. Furthermore, let $\mu(K)$ be the supremum of the $K$-widths of the parallelograms with the $T_{K}$ (3) property. It is known that $\mu(B)=\sqrt{2}$ (to see [3] and [7]), however, for an arbitrary convex body $K$ we have the following.

Theorem 1 There exists a number $\varepsilon>0$ such that for every convex body $K$,

$$
\frac{4}{3}+\varepsilon \leq \lambda(K, 3) \leq \max \left(\frac{1+\sqrt{1+4 \mu(K)}}{2}, 1.76\right) .
$$

Using this result on the disc we obtain the upper bound 1.79, which improves substantially the previous known upper bound $(\lambda(B, 3)<2)$. However, it is conjectured (to see [2]) that $\lambda(B, 3)=\frac{1+\sqrt{5}}{2}$.

\section{Corollary 1}

$$
\frac{1+\sqrt{5}}{2} \leq \lambda(B, 3) \leq \frac{1+\sqrt{1+4 \sqrt{2}}}{2} \approx 1.79004 .
$$

The following theorem establishes that the number 2 characterizes the parallelogram. 
Theorem $2 \lambda(K, 3)=2$ if and only if $K$ is a parallelogram.

Note that characterizing a class of convex bodies $[K]$ by the value of $\lambda(K, r)$ is only possible if $K$ minimizes or maximizes the value of $\lambda(K, r)$ in the BanachMazur compactum. This is because of the topology of this space; if the minimum and maximum of $\lambda(\cdot, r)$ are attained at $K_{0}$ and $K_{1}$ respectively, then there is a path in the Banach-Mazur compactum connecting $\left[K_{0}\right]$ and $\left[K_{1}\right]$ that does not pass through any other given $[K]$.

Finally, we establish the following unexpected theorem:

Theorem 3 Let $K$ be a convex body in the plane, then

$$
\lambda(K, 4) \leq \frac{1+\sqrt{5}}{2}
$$

In [8] was proved that $\lambda(B, 4)=\frac{1+\sqrt{5}}{2}$, however, it is easy to see that the number $\frac{1+\sqrt{5}}{2}$ does not characterize the Euclidean disc. Indeed, consider the regular decagon, $P$, inscribed in the unit circle $B$ and let $K$ be any convex body such that $P \subset K \subset B$ (as shown in Fig. 4).

Now, considering the pentagonal example (see Fig. 5) we have that $\lambda(K, 4) \geq$ $\frac{1+\sqrt{5}}{2}$. Then, by Theorem 3 we have that $\lambda(K, 4)=\frac{1+\sqrt{5}}{2}$.

Fig. 4 A convex body with $\lambda(K, 4)=\frac{1+\sqrt{5}}{2}$

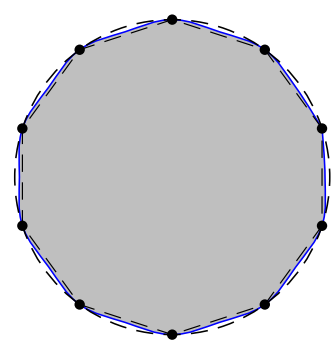

Fig. 5 Pentagonal example

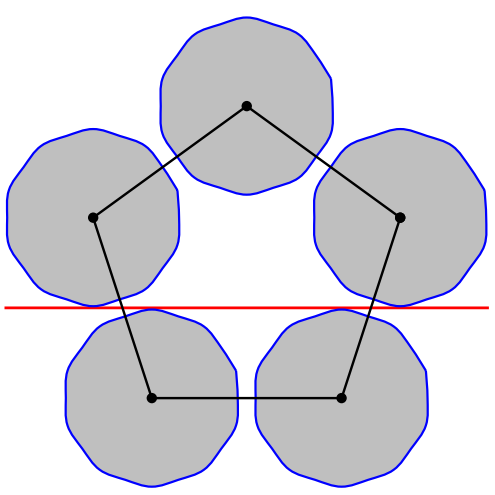




\section{Proofs}

Proof of Lemma 1 Let $\mathcal{F}=\left\{x_{1}+K_{1}, \ldots, x_{n}+K_{1}\right\}$ be a family of translates of $K_{1}$ with the $T(r)$ property. Let $\delta=\delta\left(K_{1}, K_{2}\right)$ and $\lambda=\lambda\left(K_{2}, r\right)$. We can chose an element $K \in\left[K_{2}\right]$ such that $K_{1} \subset K \subset \delta K_{1}$, and therefore the family $\left\{x_{1}+K, \ldots\right.$, $\left.x_{n}+K\right\}$ has the property $T(r)$. Since $\left\{x_{1}+\lambda K, \ldots, x_{n}+\lambda K\right\}$ has the property $T$, then $\left\{x_{1}+\lambda \delta K_{1}, \ldots, x_{n}+\lambda \delta K_{1}\right\}$ also has the property $T$. This implies $\lambda\left(K_{1}, r\right) \leq$ $\lambda \delta$.

Note. In what follows, by the area of a given set we understand its Euclidean area.

Proof of Theorem 1 To prove the first inequality, let $S$ be a square and $H$ be a regular hexagon. It is known [10] that $\delta(S, K) \leq \frac{3}{2}$ with equality if and only if $[K]=[H]$. Since $\lambda(S, 3)=2$ (see for instance [2], and [8]), we have by Lemma 1 that if $K \notin[H]$, then $\frac{4}{3}<\frac{\lambda(S, 3)}{\delta(S, K)} \leq \lambda(K, 3)$. On the other hand, since $\lambda(B, 3) \geq \frac{1+\sqrt{5}}{2}$ and $\delta(B, H)=\frac{2}{\sqrt{3}}$, we have that if $K \in[H]$, then $\frac{4}{3}<\frac{\lambda(B, 3)}{\delta(B, H)} \leq \lambda(K, 3)$. Since the application $[K] \mapsto \lambda(K, 3)$ is continuous on the Banach-Mazur compactum, the above implies that there is a number $\varepsilon>0$ such that

$$
\frac{4}{3}+\varepsilon \leq \lambda(K, 3)
$$

for all $K$.

To prove the second inequality, fix $K$ and let $X$ be a finite set of points with the $T_{K}(3)$ property.

Let $\triangle A B C$ be the triangle of biggest area formed by points in $X$ and suppose that the $K$-width of the strip bounded by the line $A C$ and its parallel through $B$ is at most 1. For simplicity we write $\mathrm{d}_{K}(P, M N)$ for the $K$-width of the strip bounded by a given line $M N$ an its parallel through $P$, so with this notation we have that $\mathrm{d}_{K}(B, A C) \leq 1$. Let $D$ be the point in the plane such that $A B C D$ is a parallelogram and let $E$ be the point in $X$ that is farthest away (in the Euclidean sense) from the line $A C$ and contained in the triangle $\triangle A C D$. Let $F$ and $G$ be points on the segments $[C, D]$ and $[A, D]$ such that $E$ is on the segment $[F, G]$ and the line $F G$ is parallel to $A C$.

Set $h_{1}=\mathrm{d}_{K}(B, A C)$ and $h_{2}=\mathrm{d}_{K}(E, A C)$. Now let $A^{\prime}, B^{\prime}$ and $C^{\prime}$ be points such that $A^{\prime} B^{\prime} C^{\prime} D$ is a parallelogram homothetic to $A B C D$ with center $D$ and ratio $\frac{h_{1}}{h_{2}}$ (see Fig. 6).

Let $H$ be any point in $X$ not contained in the parallelogram $A B C D$. It is clear that $H$ must be above the lines $C^{\prime} D$ and $A^{\prime} D$, otherwise, we were have that either $\triangle A B H$ or $\triangle B C H$ would have area greater than $|A B C|$. Without loss of generality, we may assume that $A$ and $H$ are on opposite sides of $B D$. We then have

$$
|A B C| \geq|A H E|>|A H G|=\frac{\mathrm{d}_{K}(H, A D)}{\mathrm{d}_{K}(C, A D)} \cdot \frac{\mathrm{d}_{K}(A, G)}{\mathrm{d}_{K}(A, D)} \cdot|A C D| .
$$

Notice that $\frac{\mathrm{d}_{K}(H, A D)}{\mathrm{d}_{K}(C, A D)}=\frac{\mathrm{d}(H, A D)}{\mathrm{d}(C, A D)}$ and $\frac{\mathrm{d}_{K}(A, G)}{\mathrm{d}_{K}(A, D)}=\frac{\mathrm{d}(A, G)}{\mathrm{d}(A, D)}$. Now, since $|A C D|=|A B C|$ and $\frac{\mathrm{d}_{K}(A, G)}{\mathrm{d}_{K}(A, D)}=\frac{h_{2}}{h_{1}}$, we have that $\frac{\mathrm{d}_{K}(H, A D)}{\mathrm{d}_{K}(C, A D)} \leq \frac{h_{1}}{h_{2}}$ and so $H$ is inside the triangle $\triangle D B^{\prime} C^{\prime}$. Hence the whole set $X$ is contained in the parallelogram $A^{\prime} B^{\prime} C^{\prime} D$. 
Fig. 6 All the centers are contained in $D A^{\prime} B^{\prime} C^{\prime}$

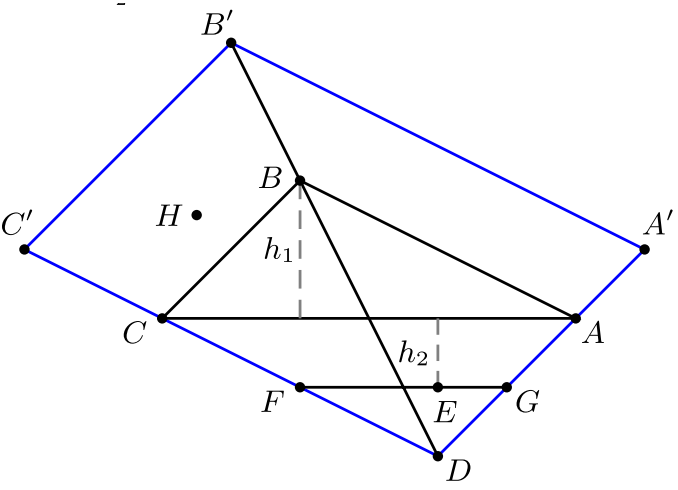

Suppose that $E$ is closer to $G$ than to $F$. The case when $E$ is closer to $F$ is analogous. We know the $K$-width of triangle $\triangle B C E$ is at most 1 , for this to happen there are three possibilities for the width of the triangle:

(1) $\mathrm{d}_{K}(C, B E) \leq 1$. In this case we have that the strip bounded by the line $B E$ and its parallel through $C$ contains the point $D$ and has $K$-width at most 1 . It follows that the triples $\{B, C, D\}$ and $\{A, B, D\}$ have the property $T_{K}(3)$. Hence $\{A, B, C, D\}$ has the $T_{K}(3)$ property and therefore the $K$-width of $A B C D$ is at most $\mu(K)$. We then obtain that the $K$-width of $A^{\prime} B^{\prime} C^{\prime} D$ is at most $\frac{h_{1}}{h_{2}} \mu(K)$.

(2) $\mathrm{d}_{K}(B, C E) \leq 1$. In this case we have that the strip bounded by the line $C E$ and its parallel through $B$ contains the segment $A G$ and has $K$-width at most 1 . It follows that the strip bounded by the lines parallel to $C E$ through $B$ and $D$ has $K$-width at most $\frac{h_{1}}{h_{2}}$. Therefore, the $K$-width of $A^{\prime} B^{\prime} C^{\prime} D$ is at most $\left(\frac{h_{1}}{h_{2}}\right)^{2}$.

(3) $\mathrm{d}_{K}(E, B C) \leq 1$. In this case we have $\mathrm{d}_{K}(D, B C)=\frac{h_{1}}{h_{2}} \cdot \mathrm{d}_{K}(F, B C) \leq \frac{h_{1}}{h_{2}}$. $\mathrm{d}_{K}(E, B C) \leq \frac{h_{1}}{h_{2}}$. Therefore the $K$-width of $A^{\prime} B^{\prime} C^{\prime} D$ is at most $\left(\frac{h_{1}}{h_{2}}\right)^{2}$.

In all three cases we have that the $K$-width of $X$ is at most

$$
\max \left\{\left(\frac{h_{1}}{h_{2}}\right)^{2}, \frac{h_{1}}{h_{2}} \mu(K)\right\} .
$$

Since $\triangle A B C$ is the biggest area triangle in $X$ and because of the definition of $E$, we have that the set $X$ has $K$-width at most $h_{1}+h_{2}$. In this way we have shown that the $K$-width of $X$ is at most the minimum of (1) and $h_{1}+h_{2}$.

The optimization problem of maximizing the minimum between (1) and $h_{1}+h_{2}$ subject to $h_{2} \leq h_{1} \leq 1$ is not difficult to solve and has a solution given by

$$
\max \left(\frac{1+\sqrt{1+4 \mu(K)}}{2}, \rho\right),
$$

where $\rho \approx 1.75488$ is the real root of the polynomial $x^{3}-2 x^{2}+x-1$. This completes the proof of the theorem. 
Fig. 7 A parallelogram with the $T_{K}$ (3)-property

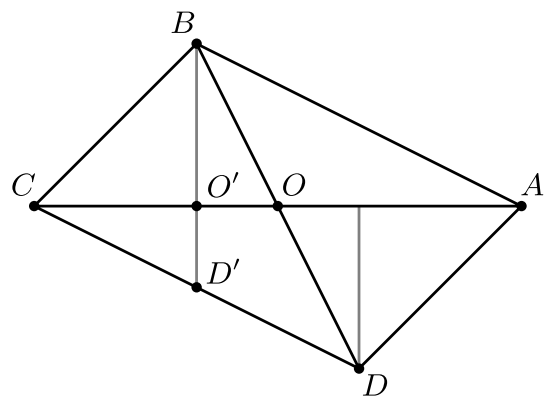

Proof of Corollary 1 The first inequality is given by the pentagonal example shown in Fig. 4. For the second inequality we use that $\mu(B)=\sqrt{2}$ (proved by J. Eckhoff [3]) and apply Theorem 1.

Proof of Theorem 2 It is known (see [3] and [8]) that if $K$ is a parallelogram, then $\lambda(K, 3)=2$. In view of Theorem 1 , it is enough to show that $\mu(K)=2$ only if $K$ is a parallelogram. First we observe that $\mu$ is actually a maximum.

Using a standard compactness argument, it is enough to prove that every parallelogram with the $T_{K}(3)$ property and big enough diameter has $K$-width close to 1 . This can be easily done using Theorem $\mathrm{H} 2$ in the following way: let $A B C D$ be such a parallelogram, if the diameter of $A B C D$ is big enough we may choose a large set of points on the diameter of $A B C D$ in such way that their mutual $K$-distances are bigger than 1 and their $K$-distances to the vertices of the parallelogram are also bigger than 1 (otherwise $A B C D$ has $K$-width 1). Clearly, these points together with the vertices of the parallelogram have the $T_{K}(3)$ property. Theorem $\mathrm{H} 2$ now implies that the $K$-width of $A B C D$ is $1+w / 2$, for a small enough $w>0$. This implies that $\mu$ is actually a maximum and therefore we can always find a parallelogram with the $T_{K}(3)$ property and $K$-width equal to $\mu(K)$.

Now, let $X=\{A, B, C, D\}$ be the vertex set of a parallelogram with the property $T_{K}$ (3) and $K$-width equal to 2. Let $O$ be the center of the parallelogram (as shown in Fig. 7). We have that the $K$-width of triangle $\triangle A B C$ must be realized using the vertex $B$, otherwise the $K$-width of $X$ would be at most 1 . Let $O^{\prime}$ be a point on the segment $[C, A]$ such that $\mathrm{d}_{K}\left(B, O^{\prime}\right)=1$. Clearly this point exists, otherwise, we would have that the $K$-width of $A B C D$ would be less than 1 . Without loss of generality suppose that $O^{\prime}$ is contained in the segment $[C, O]$. Now, let $D^{\prime}$ be the intersection of the lines $B O^{\prime}$ and $C D$. If $O \neq O^{\prime}$, then $\mathrm{d}_{K}\left(B, D^{\prime}\right)<2$ and the $K$-width of $X$ would be less than 2. So, $O=O^{\prime}$ and therefore $\mathrm{d}_{K}(O, B)=1$. Analogously, we have that $\mathrm{d}_{K}(O, A)=1$. Since the $K$-width of $X$ is $2, \mathrm{~d}_{K}(O, P) \geq 1$ for any point $P$ in the boundary of the parallelogram. The convexity of $K$ implies that $\mathrm{d}_{K}(O, P) \leq 1$ for any point $P$ in the boundary of the parallelogram. It follows that $\mathrm{d}_{K}(O, P)=1$ for every point in the boundary of the parallelogram. Therefore $K$ is the parallelogram $A B C D$. 
Fig. 8 Triangle with maximum area

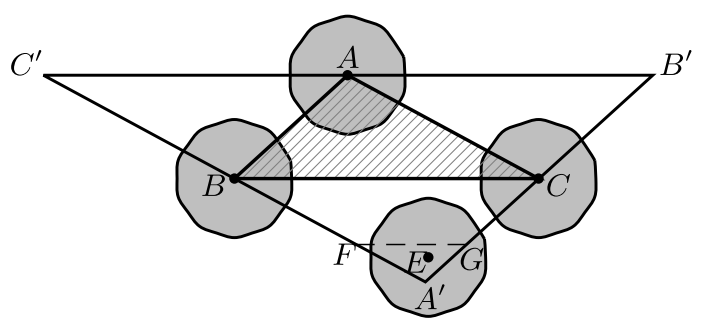

Fig. 9 Triangle with maximum area inscribed in a pentagon

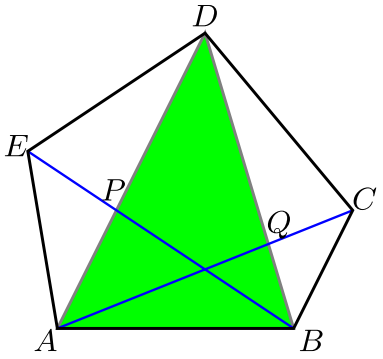

Proof of Theorem 3 The proof of Theorem 1 given in [8] works for a general convex body $K$ if we replace the Euclidean width by the $K$-width. So, we will follow the main idea in [8] with some simple modifications.

Consider a fixed convex body $K$. By the observations given before, we may consider that $K$ is a centrally symmetric set. Let $\mathcal{F}$ be a finite family of translates of $\frac{1}{2} K$ with the $T(4)$ property and let $X$ be the set consisting on the centers of the translates. We have that $X$ has the $T_{K}$ (4) property. Also, let $A, B, C \in X$ be points such that the area of $\triangle A B C$ is maximal. Clearly, $X \subset \triangle A^{\prime} B^{\prime} C^{\prime}$, where $\triangle A^{\prime} B^{\prime} C^{\prime}$ is the triangle homothetic to $\triangle A B C$ with center of homothecy at the centroid of $\triangle A B C$ and constant of homothecy equal to -2 (see Fig. 8). Suppose that $\mathrm{d}_{K}(A, B C) \leq 1$ and let $E \in X$ be the point in $\triangle A^{\prime} B^{\prime} C^{\prime}$ which is further apart from the line $C^{\prime} B^{\prime}$. We may also assume that $\mathrm{d}_{K}\left(E, C^{\prime} B^{\prime}\right)>\tau=\frac{1+\sqrt{5}}{2}$, otherwise we are done.

Now we will give the statement of a lemma due to E.G. Straus [9] whose direct implications are an important ingredient in the proof of the theorem. We shall return to the proof of Theorem 3 soon after.

Lemma $\mathbf{S}$ Let $\Gamma$ be a convex body in the plane and assume that one of the sides of an inscribed triangle $\Delta$ of maximum area lies on bd $\Gamma$, then the ratio of the areas satisfies $\sqrt{5} \cdot|\Delta| \geq|\Gamma|$, with equality if and only if $\Gamma$ is an affine regular pentagon.

It is easy to see that when $\Gamma$ is a convex pentagon then there is a triangle, $\Delta$, of maximum area whose vertices are vertices of the pentagon. Hence, one of the sides of $\Delta$ lies on bd $\Gamma$ and so Straus's lemma applies. Suppose that $A, B, C, D, E$ are the vertices of $\Gamma$ and $\triangle A B D$ is a triangle with maximum area. Let $P$ be the point where $[A, D]$ intersects $[B, E]$ and let $Q$ be the point where $[A, C]$ intersects $[B, D]$ (as shown in Fig. 9). 
Fig. 10 All the centers are in the shaded region

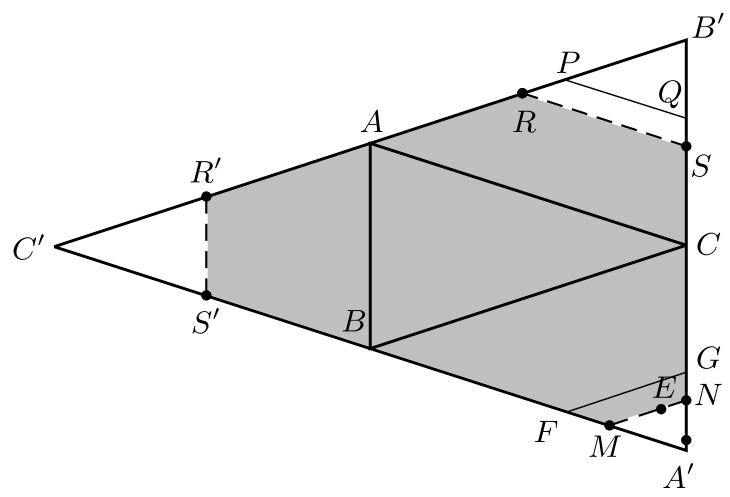

Claim $1 \frac{\mathrm{d}(E, B)}{\mathrm{d}(P, B)}+\frac{\mathrm{d}(A, C)}{\mathrm{d}(A, Q)} \leq 2 \tau$

Proof of Claim 1 By the maximality of $|A B D|$ and by Lemma $\mathrm{S}$ we have that $|D B C|+$ $|D A E|+|A B D| \leq \sqrt{5} \cdot|A B D|$. In other words

$$
\frac{\frac{\mathrm{d}(Q, C)}{\mathrm{d}(A, Q)} \cdot|A B D|+\frac{\mathrm{d}(E, P)}{\mathrm{d}(P, B)} \cdot|A B D|+|A B D|}{|A B D|} \leq \sqrt{5},
$$

that is

$$
\frac{\mathrm{d}(Q, C)}{\mathrm{d}(A, Q)}+\frac{\mathrm{d}(E, P)}{\mathrm{d}(P, B)}+1 \leq \sqrt{5}
$$

It follows that

$$
\frac{\mathrm{d}(Q, C)}{\mathrm{d}(A, Q)}+1+\frac{\mathrm{d}(E, P)}{\mathrm{d}(P, B)}+1 \leq 1+\sqrt{5},
$$

therefore, $\frac{\mathrm{d}(Q, C)+\mathrm{d}(A, Q)}{\mathrm{d}(A, Q)}+\frac{\mathrm{d}(E, P)+\mathrm{d}(P, B)}{\mathrm{d}(P, B)}=\frac{\mathrm{d}(A, C)}{\mathrm{d}(A, Q)}+\frac{\mathrm{d}(E, B)}{\mathrm{d}(P, B)} \leq 1+\sqrt{5}$.

Notice that Claim 1 remains true if instead of Euclidean distance we consider the $K$-distance.

Now, we return to the proof of Theorem 3. Let $M \in\left[B, A^{\prime}\right]$ and $N \in\left[C, A^{\prime}\right]$ such that $E \in[M, N]$ and $M N \| B C$ (see Fig. 10). Consider the points $R, P \in\left[A, B^{\prime}\right]$ and $Q, S \in\left[C, B^{\prime}\right]$ such that $\frac{\mathrm{d}_{K}\left(R, C^{\prime} A^{\prime}\right)}{\mathrm{d}_{K}\left(A, C^{\prime} A^{\prime}\right)}+\frac{\mathrm{d}_{K}\left(M, C^{\prime} B^{\prime}\right)}{\mathrm{d}_{K}\left(B, C^{\prime} B^{\prime}\right)}=2 \tau$, and $\frac{\mathrm{d}_{K}\left(P, C^{\prime} A^{\prime}\right)}{\mathrm{d}_{K}\left(A, C^{\prime} A^{\prime}\right)}=\tau$. Similarly, we define the points $R^{\prime} \in\left[C^{\prime}, A\right]$ and $S^{\prime} \in\left[C^{\prime}, B\right]$ such that $R^{\prime} S^{\prime} \| A B$ and $\frac{\mathrm{d}_{K}\left(S^{\prime}, B^{\prime} A^{\prime}\right)}{\mathrm{d}_{K}\left(B, B^{\prime} A^{\prime}\right)}+\frac{\mathrm{d}_{K}\left(M, C^{\prime} B^{\prime}\right)}{\mathrm{d}_{K}\left(B, C^{\prime} B^{\prime}\right)}=2 \tau$. By Claim 1 we know that $X$ must be contained in the hexagon $R R^{\prime} S^{\prime} M N S$. It follows that if either $\mathrm{d}_{K}(B, A C) \leq 1$ or $\mathrm{d}_{K}(C, A B) \leq 1$ then either the strip bounded by $R S$ and $C^{\prime} A^{\prime}$ or the strip bounded by $R^{\prime} S^{\prime}$ and $B^{\prime} A^{\prime}$ has $K$-width less than or equal to $\tau$. Then, we may assume now that $\mathrm{d}_{K}(B, A C)>1$ and $\mathrm{d}_{K}(C, A B)>1$.

Since $\{A, B, C, E\} \in T_{K}(4)$ we have that either $\mathrm{d}_{K}(A, B E) \leq 1$ or $\mathrm{d}_{K}(A, C E) \leq 1$. We will consider that $\mathrm{d}_{K}(A, B E) \leq 1$, the other case is completely analogous. Now, let $S_{1}$ be the strip bounded by the line $B E$ and its parallel through $A$ and let $S_{2}$ be 
Fig. 11 All the centers are between $\ell_{2}$ and $\ell_{1}$

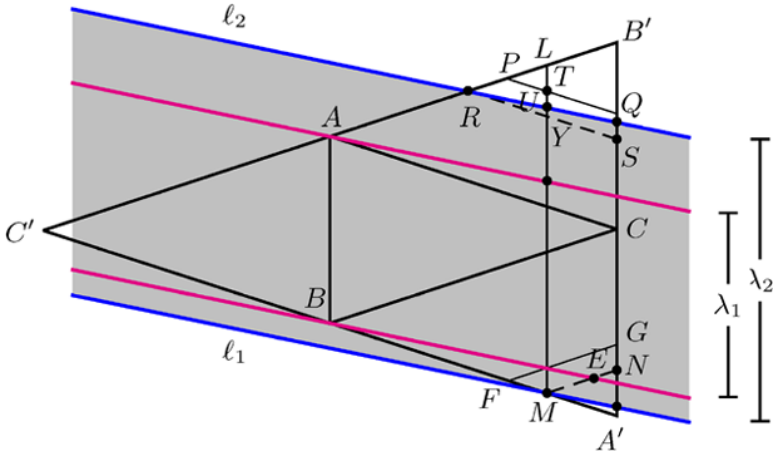

the strip bounded by the two lines parallel to $B E$ through the points $R$ and $M$, respectively. Let $\lambda_{1}$ and $\lambda_{2}$ be the $K$-width of $S_{1}$ and $S_{2}$, respectively; clearly $\lambda_{1} \leq 1$ and we will prove that $\lambda_{2} \leq \tau$ (see Fig. 11). Let $T \in[P, Q]$ be the point such that $M T \| A B$, and let $Y$ and $L$ be the points where the line $M T$ intersects the segments $[R, S]$ and $\left[R, B^{\prime}\right]$, respectively.

Since $\frac{\mathrm{d}_{K}(F, M)}{\mathrm{d}_{K}\left(B, A^{\prime}\right)}=\frac{\mathrm{d}_{K}(R, P)}{\mathrm{d}_{K}\left(A, B^{\prime}\right)}$ we have that $\mathrm{d}_{K}(P, L)=\mathrm{d}_{K}(R, P)$, hence $T$ is the midpoint of the segment $[L, Y]$ and then the line $R T$ intersects the segment $\left[B^{\prime}, S\right]$ at its midpoint. It follows that $\ell_{2}$ intersects the interior of $[T, Y]$. We have that

$$
\frac{\mathrm{d}_{K}(U, M)}{\mathrm{d}_{K}(A, B)}<\frac{\mathrm{d}_{K}(T, M)}{\mathrm{d}_{K}(A, B)}=\frac{\mathrm{d}_{K}\left(Q, A^{\prime}\right)}{\mathrm{d}_{K}(A, B)}=\frac{1+\sqrt{5}}{2} ;
$$

this implies that $\frac{\lambda_{2}}{\lambda_{1}}<\frac{1+\sqrt{5}}{2}$. Therefore, $\lambda_{2}<\frac{1+\sqrt{5}}{2}$.

Acknowledgements We thank, for support and hospitality, the Alfréd Rényi Mathematics Institute. We also thank to I. Bárány, J. Eckhoff, A. Heppes, G. Fejes Tóth, and G. Ambrus for many helpful and inspiring discussions on problems about line transversals.

\section{References}

1. Ageev, S., Repovŝ, D.: On Banach-Mazur compacta. J. Aust. Math. Soc., Ser. A 69, 316-335 (2000)

2. Eckhoff, J.: Transversalenprobleme vom Gallai'schen Typ. Dissertation, Göttingen (1969)

3. Eckhoff, J.: Problem 11247. Am. Math. Mon. 113, 760 (2006)

4. Grünbaum, B.: On common transversals. Arch. Math. 9, 465-469 (1958)

5. Heppes, A.: New upper bound on the transversal width of T(3)-families of discs. Discrete Comput. Geom. 34, 463-474 (2005)

6. Heppes, A.: The width of the transversal strips of $T$ (3)-families in the plane. Discrete Comput. Geom. 34, 455-461 (2005)

7. Huicochea, M., Jerónimo-Castro, J.: The strip of minimum width covering a centrally symmetric set of points. Period. Math. Hung. 58, 47-58 (2009)

8. Jerónimo, J.: Line transversals to translates of unit discs. Discrete Comput. Geom. 37, 409-417 (2007)

9. Straus, E.G.: Some Extremal Problems in Combinatorial Geometry. Lecture Notes in Math., vol. 686, 308-312. Springer, Berlin (1978)

10. Stromquist, W.: The maximum distance between two-dimensional Banach spaces. Math. Scan 48, 205-225 (1981)

11. Tverberg, H.: Proof of Grünbaum's conjecture on common transversals for translates. Discrete Comput. Geom. 4, 191-203 (1989) 